\title{
Квантование электромагнитного поля в трехмерных фотонных структурах на основе формализма матрицы рассеяния ( $S$-квантование)
}

\author{
(C) К.А. Иванов ${ }^{1}$, А.Р. Губайдуллин ${ }^{1,3}$, М.А. Калитеевский ${ }^{1,2,3, \text { ฯ }}$ \\ ${ }^{1}$ Санкт-Петербургский национальный исследовательский университет \\ информационных технологий, механики и оптики, \\ 197101 Санкт-Петербург, Россия \\ ${ }^{2}$ Физико-технический институт им. А.Ф. Иоффе Российской академии наук, \\ 194021 Санкт-Петербург, Россия \\ ${ }^{3}$ Санкт-Петербургский Академический университет, \\ 194021 Санкт-Петербург, Россия \\ ฯ E-mail: m.kaliteevski@mail.ru; kivanov@corp.imfo.ru
}

(Получена 11 декабря 2017 г. Принята к печати 14 декабря 2017 г.)

\begin{abstract}
На основе формализма матрицы рассеяния развит метод квантования электромагнитного поля в фотонных наноструктурах с трехмерной модуляцией диэлектрической проницаемости $(S$-квантование в трехмерном случае). Квантование основано на приравнивании единице собственных чисел матрицы рассеяния, что эквивалентно приравниванию друг другу совокупности полей, раскладываемых в ряды Фурье, падающих на структуру волн и убегающих от структуры волн. В фотонной наноструктуре пространственное изменение мод электромагнитного поля рассчитано на основе матриц $\hat{R}$ и $\hat{T}$, описывающих отражение и прохождение компонент Фурье через структуру. Для вычисления коэффициентов отражения и пропускания отдельных координатных и фурье-компонент структура разбивается на параллельные слои, в которых диэлектрическая проницаемость меняется в двумерном пространстве. При помощи преобразования Фурье уравнения Максвелла записываются в виде матрицы, связывающей компоненты Фурье электрического поля на границах соседних слоев. На основе вычисленных векторов отражения и пропускания для всех поляризаций и Фурье-компонент формируется матрица рассеяния для всей структуры и осуществляется квантование путем приравнивания единице собственных чисел матрицы рассеяния. Развитый метод позволяет получать пространственные профили собственных мод без решения системы нелинейных интегро-дифференциальных уравнений и значительно сокращает вычислительные ресурсы, необходимые для расчета вероятности спонтанного излучения в трехмерных системах.
\end{abstract}

DOI: 10.21883/FTP.2018.09.46150.8796

\section{1. Введение}

Описание взаимодействия излучения с веществом [1] является одной из важных задач современной физики, интерес обусловлен возможностью создания оптоэлектронных устройств с настраиваемыми характеристиками. Известно, что среды с наличием неоднородности диэлектрической проницаемости позволяют контролировать направление и вероятность спонтанного излучения [2]. В частности, вероятность спонтанного излучения может существенно возрастать для собственной моды резонатора [3]. В средах, в которых пространственное распределение диэлектрической проницаемости изменяется периодически и наблюдается брэгговская дифракция света, формируется фотонная запрещенная зона, в которой спонтанное излучение может быть полностью подавлено [4,5]. В последние годы популярны исследования наноструктур, устроенных особым способом [6], обладающих интересными свойствами: в частности в резонаторах металл/полупроводник с трехмерной модуляцией диэлектрической проницаемости получена лазерная генерация [7]. Интерес вызывают системы, имеющие сложную структуру фотонных зон [8], потенциально применимые в терагерцовом диапазоне.
Для описания взаимодействия излучения с веществом разработана процедура квантования электромагнитного поля в однородном „ящике квантования“, которая позволяет осуществлять переход от непрерывного спектра частот к квазинепрерывному. Процедура квантования подразумевает постановку на границах „ящика квантования“ граничных условий (ГУ), традиционным выбором являются периодические граничные условия БорнаКармана $[1,9,10]$. Для вычисления вероятности спонтанного излучения в слоистых структурах предложен метод, основанный на изменении пространственной структуры мод электромагнитного поля [11]. Однако в случае неоднородной среды метод исследования модовой структуры поля на основе периодических ГУ не является строгим и самосогласованным, поскольку наличие неоднородности может приводить к существенному изменению модовой структуры, рассчитанной на основе периодических ГУ, что может приводить к неточному результату при исследовании систем конечного размера [12]. Численные алгоритмы расчета вероятности спонтанной эмиссии на основе функции Грина [13] или на основе разложения поля по собственным модам, квазистационарным состояниям [14], при реализации для трехмерных систем большого размера требуют значительных вычислитель- 
ных ресурсов и могут сопровождаться проблемами со сходимостью результатов.

Построение строгой и самосогласованной процедуры квантования электромагнитного поля и вычисления вероятности спонтанной эмиссии в неоднородных средах может быть реализовано на основе формализма $S$-квантования [15], в котором условием квантования считается эквивалентность амплитуд волн, падающих на структуру с амплитудами убегающих волн.

Данная работа посвящена развитию формализма $S$-квантования для сред с трехмерной модуляцией диэлектрической проницаемости.

\section{2. Квантование электромагнитного поля}

Рассмотрим произвольную структуру с трехмерными неоднородностями. Необходимо выбрать в ней систему координат. Поскольку в большинстве создаваемых в настоящее время структур можно выделить одну ось (ось роста), выберем ее в качестве оси $z$; при этом толщина структуры вдоль этой оси (обозначим ее $L_{z}$ ) обычно значительно меньше поперечных размеров структуры в плоскости $x y$. Предположим, что характерные поперечные размеры структуры в этой плоскости составляют $L_{x} \times L_{y}$ (см. рисунок). Это дает возможность разложить все величины, имеющиеся в задаче квантования поля, в ряд Фурье по двум измерениям. Например, электрическое поле может быть представлено в виде

$$
\mathbf{E}(x, y, z)=\sum_{\mathbf{q}} \mathbf{E}(z)_{\mathbf{q}} \exp (i \mathbf{q} \boldsymbol{\rho})
$$

В этой записи компоненты двумерного вектора $\mathbf{q}=\left(q_{x}, q_{y}\right)$ пробегают значения $2 \pi M_{x, y} / L_{x, y}$, где $M_{x, y}$ - целые числа, а вектор $\rho$ лежит в плоскости $x y$. Сразу понятно, что такое преобразование, вообще говоря, не допускает произвольных значений для угла падения какой-либо волны на структуру. Однако можно выбрать достаточно большие поперечные размеры структуры, так что поперечный волновой вектор будет близок к одному из векторов q, если только количество членов в разложении будет достаточным. В частности, для волн оптического диапазона достаточно, чтобы поперечный размер структуры превышал 100 мкм.

Кроме того, благодаря линейности уравнений Максвелла любую волну, падающую на структуру, можно разложить на две поляризации: с нулевой компонентой $E_{x}$ или $E_{y}$. Отметим, что эти поляризации не имеют ничего общего с ТЕ- или ТМ-поляризацией, так как плоскость падения отсутствует как таковая.

Как будет показано в следующем разделе, $x$ - и $y$-компоненты электрического и магнитного полей однозначно определяют решение уравнений Максвелла, поэтому достаточно определить вектор коэффициентов Фурье $x$ - и $y$-компонент электрического поля, чтобы решить задачу квантования. Далее в этом разделе везде,

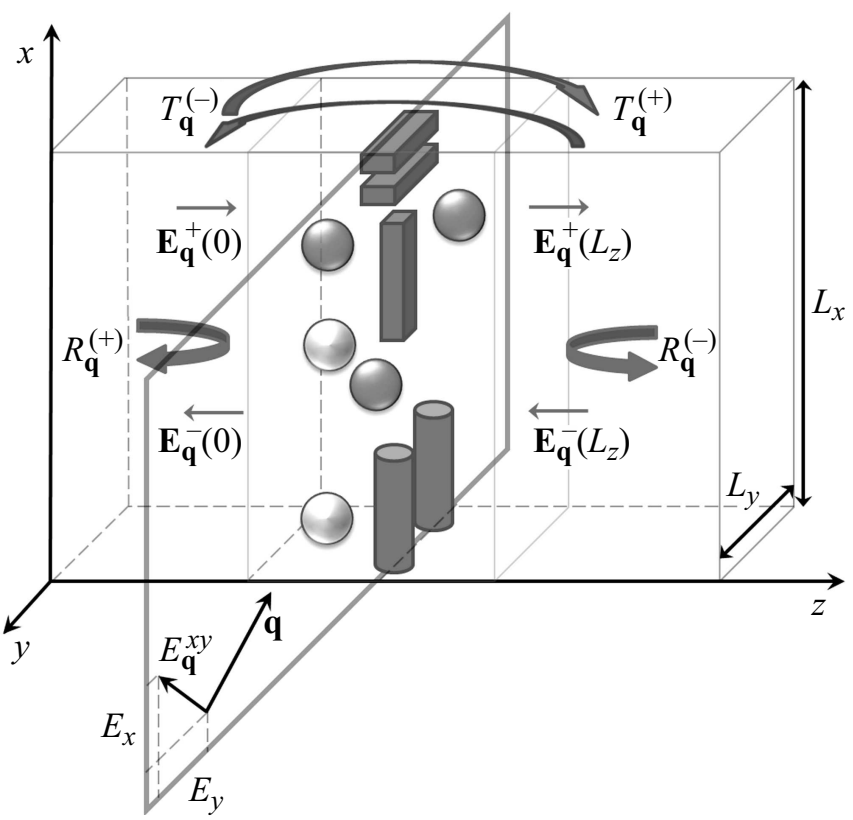

Схема структуры, помещенной в ящик квантования.

где в матричной записи встречается индекс q, подразумевается, что он дважды пробегает весь набор значений в фурье-пространстве, по одному разу для каждой из координатных компонент электрического поля.

Процедура $S$-квантования основана на построении матрицы рассеяния, связывающей амплитуды волн, падающих на структуру, с амплитудами волн, испускаемых структурой. Таким образом,

$$
\begin{gathered}
\left(\begin{array}{c}
E_{\mathbf{q}}^{(+)}\left(L_{z}\right) \\
E_{\mathbf{q}}^{(-)}(0)
\end{array}\right)=\hat{S} \times\left(\begin{array}{c}
E_{\mathbf{q}}^{(+)}(0) \\
E_{\mathbf{q}}^{(-)}\left(L_{z}\right)
\end{array}\right), \\
\hat{S}=\left(\begin{array}{cc}
\hat{T}^{(+)} & \hat{R}^{(-)} \\
\hat{R}^{(+)} & \hat{T}^{(-)}
\end{array}\right) .
\end{gathered}
$$

Здесь введены следующие обозначения: верхний индекс обозначает направление распространения волны по оси $z$ (положительное или отрицательное), а матрицы $\hat{T}$ и $\hat{R}$ описывают прохождение и отражение компонент Фурье через структуру. Например, если существует только волна, распространяющаяся в положительном направлении оси $z$, то можно записать

$$
E_{\mathbf{q}}^{(+)}\left(L_{z}\right)=\hat{T}_{\mathbf{q}, \mathbf{q}^{\prime}}^{(+)} E_{\mathbf{q}^{\prime}}^{(+)}(0) .
$$

Способ вычисления матриц отражения и прохождения представлен в следующем разделе.

Процедура $S$-квантования подразумевает приравнивание собственных чисел матрицы рассеяния единице:

$$
\begin{gathered}
\|\hat{S}-\lambda(\omega) \hat{I}\|=0, \\
\lambda(\omega)=1 .
\end{gathered}
$$

Таким образом, получим собственные моды электромагнитного поля для данной структуры. Каждому собственному числу соответствует собственный вектор, 
вычислив который, получаем исходные значения поля на границах. Далее, используя метод матриц переноса, рассмотренный в следующем разделе, можно восстановить структуру поля для данной моды в любой точке структуры. Пользуясь распределением поля, можно вычислить такие величины, как вероятность спонтанного дипольного излучения и, как следствие, фактор Парселла [12,15].

\section{3. Вычисление коэффициентов отражения и прохождения}

Для нахождения матриц отражения $\hat{R}^{( \pm)}$и пропускания $\hat{T}^{( \pm)}$(3) существуют различные способы [16], обычно использующие метод конечных разностей. Недостатком таких методов является прежде всего вычислительная сложность, особенно при работе в прямом пространстве. При работе в обратном пространстве также возникают расходимости для некоторых типов материалов. В одномерных структурах имеет смысл пользоваться методом матриц переноса [17], который хорошо подходит для вычисления коэффициентов отражения и пропускания и уже был использован в методике $S$-квантования низших размерностей $[12,15,18]$. В данной работе предложен обобщенный метод матриц переноса для случая трехмерных структур, удовлетворяющих требованиям, перечисленным в предыдущем разделе. В этом разделе сохраняется соглашение по поводу индекса q в матричной записи, однако теперь он пробегает значения из обратного пространства четырежды: для $x$ и $y$-компонент электрического и магнитного полей.

В основе метода лежит разбиение структуры на $N$ слоев, параллельных плоскости $x y$. Толщину слоя с номером $l$ обозначим через $d_{l}$. Внутри каждого слоя положим диэлектрическую проницаемость $\varepsilon$ зависящей только от $x$ и $y$,

$$
\varepsilon=f(x, y),
$$

и разложим ее в ряд Фурье аналогично полю в формуле (1):

$$
\varepsilon(x, y)=\sum_{\mathbf{q}} \varepsilon_{\mathbf{q}} \exp (i \mathbf{q} \boldsymbol{\rho})
$$

Отметим, что с ростом абсолютной величины $q$ коэффициенты Фурье будут стремиться к нулю, и именно это дает возможность перейти от бесконечного набора фурье-компонент поля к конечному. Тогда по теореме о свертке можно найти разложение для электрической индукции:

$$
\mathbf{D}_{\mathbf{q}}(z)=\sum_{\mathbf{q}^{\prime}} \varepsilon_{\mathbf{q}-\mathbf{q}^{\prime}} \mathbf{E}_{\mathbf{q}^{\prime}}(z) .
$$

Уравнения Максвелла для поля внутри слоя

$$
\begin{gathered}
\nabla \times \mathbf{E}=-\frac{i \omega}{c} \mathbf{H}, \quad \nabla \times \mathbf{H}=\frac{i \omega}{c} \mathbf{D}, \\
\nabla \cdot \mathbf{E}=\nabla \cdot \mathbf{H}=0
\end{gathered}
$$

преобразуются после взятия ротора от (10) и подстановки (9) и (11) в систему однородных дифференциальных уравнений 2-го порядка для фурье-компонент:

$$
\begin{gathered}
\Delta \mathbf{E}_{\mathbf{q}}(z)+\frac{\omega^{2}}{c^{2}} \sum_{\mathbf{q}^{\prime}} \varepsilon_{\mathbf{q}-\mathbf{q}^{\prime}} \mathbf{E}_{\mathbf{q}^{\prime}}(z)=0, \\
\Delta \mathbf{H}_{\mathbf{q}}(z)+\frac{\omega^{2}}{c^{2}} \sum_{\mathbf{q}^{\prime}} \varepsilon_{\mathbf{q}-\mathbf{q}^{\prime}} \mathbf{H}_{\mathbf{q}^{\prime}}(z)=0 .
\end{gathered}
$$

Оператор Лапласа в выбранном фурье-представлении записывается как

$$
\Delta=\left(-q_{x}^{2},-q_{y}^{2}, \frac{d^{2}}{d z^{2}}\right) .
$$

Применяя его к (12), (13), получим систему для компонент электрического и магнитного полей:

$$
\begin{aligned}
& E_{x, \mathbf{q}}^{\prime \prime}=q^{2} E_{x, \mathbf{q}}-\frac{\omega^{2}}{c^{2}} \sum_{\mathbf{q}^{\prime}} \varepsilon_{\mathbf{q}-\mathbf{q}^{\prime}} E_{x, \mathbf{q}^{\prime}}, \\
& E_{y, \mathbf{q}}^{\prime \prime}=q^{2} E_{y, \mathbf{q}}-\frac{\omega^{2}}{c^{2}} \sum_{\mathbf{q}^{\prime}} \varepsilon_{\mathbf{q}-\mathbf{q}^{\prime}} E_{y, \mathbf{q}^{\prime}}, \\
& H_{x, \mathbf{q}}^{\prime \prime}=q^{2} H_{x, \mathbf{q}}-\frac{\omega^{2}}{c^{2}} \sum_{\mathbf{q}^{\prime}} \varepsilon_{\mathbf{q}-\mathbf{q}^{\prime}} H_{x, \mathbf{q}^{\prime}}, \\
& H_{y, \mathbf{q}}^{\prime \prime}=q^{2} H_{y, \mathbf{q}}-\frac{\omega^{2}}{c^{2}} \sum_{\mathbf{q}^{\prime}} \varepsilon_{\mathbf{q}-\mathbf{q}^{\prime}} H_{y, \mathbf{q}^{\prime}} .
\end{aligned}
$$

При этом уравнения для $y$-компонент не нужны (см. далее). Такая система решается стандартным способом: при помощи введения вспомогательных функций первых производных по z; тогда вектор неизвестных примет вид

$$
\mathbf{x}(z)=\left(E_{x, \mathbf{q}}, E_{y, \mathbf{q}}, H_{x, \mathbf{q}}, H_{y, \mathbf{q}}, E_{x, \mathbf{q}}^{\prime}, E_{y, \mathbf{q}}^{\prime}, H_{x, \mathbf{q}}^{\prime}, H_{y, \mathbf{q}}^{\prime}\right),
$$

а система -

$$
\begin{gathered}
\mathbf{x}^{\prime}(z)=\mathbf{A x}(z), \\
\mathbf{A}=\left(\begin{array}{cc}
0 & \delta_{\mathbf{q}, \mathbf{q}^{\prime}} \\
q^{2} \delta_{\mathbf{q}, \mathbf{q}^{\prime}}-\frac{\omega^{2}}{c^{2}} \varepsilon_{\mathbf{q}-\mathbf{q}^{\prime}} & 0
\end{array}\right) .
\end{gathered}
$$

Введем координаты границ слоя $z_{1}=\sum_{j=1}^{l-1} d_{j}$ и $z_{2}=\sum_{j=1}^{l} d_{j}$ $=z_{1}+d_{l}$. Тогда можно записать решение $(20)$ в виде

$$
\mathbf{x}\left(z_{2}-0\right)=e^{d_{l} \mathbf{A}} \mathbf{x}\left(z_{1}+0\right) .
$$

Необходимо еще определить правило для переноса вектора неизвестных через границу. Используя уравнения (10), получим

$$
\begin{gathered}
E_{x, \mathbf{q}}^{\prime}=i q_{x} E_{z, \mathbf{q}}-i \frac{\omega}{c} H_{y, \mathbf{q}}, \\
E_{x, \mathbf{q}}^{\prime}=i q_{y} E_{z, \mathbf{q}}+i \frac{\omega}{c} H_{x, \mathbf{q}}, \\
H_{y, \mathbf{q}}^{\prime}=i q_{x} H_{z, \mathbf{q}}+i \frac{\omega}{c} \sum_{\mathbf{q}^{\prime}} \varepsilon_{\mathbf{q}-\mathbf{q}^{\prime}} E_{y, \mathbf{q}^{\prime}},
\end{gathered}
$$




$$
\begin{gathered}
H_{y, \mathbf{q}}^{\prime}=i q_{y} H_{z, \mathbf{q}}-i \frac{\omega}{c} \sum_{\mathbf{q}^{\prime}} \varepsilon_{\mathbf{q}-\mathbf{q}^{\prime}} E_{x, \mathbf{q}^{\prime}}, \\
\frac{\omega}{c} \sum_{\mathbf{q}^{\prime}} \varepsilon_{\mathbf{q}-\mathbf{q}^{\prime}} E_{z, \mathbf{q}^{\prime}}=q_{x} H_{y, \mathbf{q}}-q_{y} H_{x, \mathbf{q}}, \\
\frac{\omega}{c} H_{z, \mathbf{q}}==q_{y} E_{x, \mathbf{q}}-q_{x} E_{y, \mathbf{q}} .
\end{gathered}
$$

Видно, что можно вовсе избавиться от $y$-компонент полей, выразив их через остальные компоненты, а затем и выразить производные через эти компоненты. Поскольку тангенциальные проекции полей сохраняются на границах раздела сред, сохраняются и фурье-компоненты $E_{x, \mathbf{q}}, E_{y, \mathbf{q}}, H_{x, \mathbf{q}}, H_{y, \mathbf{q}}$, поэтому перенос для вектора х записывается следующим образом:

$$
\begin{gathered}
\mathbf{x}\left(z_{1}+0\right)=\mathbf{B} \mathbf{x}\left(z_{1}-0\right), \\
\mathbf{B}=\left(\begin{array}{cc}
\delta_{\mathbf{q}, \mathbf{q}^{\prime}} & 0 \\
\mathbf{C} & 0
\end{array}\right) .
\end{gathered}
$$

Введем теперь матрицу переноса через слой $l$,

$$
\hat{V}_{l}=e^{d_{l} \mathbf{A}_{l}} \mathbf{B}_{l},
$$

и матрицу переноса через всю структуру,

$$
\hat{V}=\prod_{l=1}^{N} \hat{V}_{l}
$$

При помощи такой матрицы можно рассчитать прохождение и отражение произвольной волны, падающей на любую границу структуры. Поскольку для матрицы рассеяния важны коэффициенты отражения и пропускания для отдельных координатных и фурье-компонент, покажем, как их можно вычислить. Для этого запишем волновой вектор одной компоненты

$$
\begin{gathered}
\mathbf{k}_{\mathbf{q}}=\left(q_{x}, q_{y}, \sqrt{\omega^{2} / c^{2}-q^{2}}\right), \\
\mathbf{n}_{\mathbf{q}}=\mathbf{k}_{\mathbf{q}} / k_{\mathbf{q}} .
\end{gathered}
$$

Тогда, например, для поляризации вдоль $x$

$$
\begin{gathered}
\mathbf{E}_{\mathbf{q}}=\mathbf{e}_{y} \times \mathbf{n}_{\mathbf{q}}, \\
\mathbf{H}_{\mathbf{q}}=\left(\mathbf{e}_{y} \times \mathbf{n}_{\mathbf{q}}\right) \times \mathbf{n}_{\mathbf{q}} .
\end{gathered}
$$

Этих соотношений достаточно для того, чтобы составить векторы $\mathbf{x}$, соответствующие падающей, отраженной и прошедшей волнам, поскольку зависимость от $z$ в вакууме дается обычным экспоненциальным множителем.

Запишем, как выглядят компоненты вектора х (обозначим такой вектор $\mathbf{1}_{\mathbf{q}}^{(+)}$, соответствующие компоненте Фурье q, положительному направлению распространения волны и поляризации вдоль $x$ (поляризация вдоль $y$ рассматривается аналогично), опуская индекс q:

$$
E_{x}=\sqrt{1-\frac{q^{2}}{\omega^{2} / c^{2}}}, E_{y}=0,
$$

$$
\begin{gathered}
H_{x}=\frac{q_{x} q_{y}}{\omega^{2} / c^{2}}, H_{y}=\frac{q_{y}^{2}}{\omega^{2} / c^{2}}-1, \\
E_{x}^{\prime}=i \frac{\omega}{c}\left(1-\frac{q^{2}}{\omega^{2} / c^{2}}\right), E_{y}^{\prime}=0, \\
H_{x}^{\prime}=i \frac{q_{x} q_{y}}{\omega / c} \sqrt{1-\frac{q^{2}}{\omega^{2} / c^{2}}}, \\
H_{y}^{\prime}=i\left(\frac{q_{y}^{2}}{\omega^{2} / c^{2}}-1\right) \sqrt{\omega^{2} / c^{2}-q^{2}} .
\end{gathered}
$$

Отметим, что при замене направления распространения на противоположное (в сторону убывания $z$ ) поменяют знаки компоненты $E_{x}, E_{y}$ и производные $H_{x}^{\prime}, H_{y}^{\prime}$. Таким образом, чтобы записать вектор неизвестных $\mathbf{R}_{\mathbf{q}}^{(+)}$, соответствующий столбцу матрицы отражения из (3), нужно умножить компоненты, соответствующие магнитному полю и производной электрического поля из уравнений (38), (39), на $R_{\mathbf{q}, x}$, а компоненты электрического поля и производной магнитного поля из $(37),(40)$, на $\left(-R_{\mathbf{q}, x}\right)$, поскольку отраженная волна распространяется в направлении, обратном падающей. Вектор прошедшей волны $\tilde{T}_{\mathbf{q}}^{(+)}$составляется простым умножением $(37)-(40)$ на $T_{\mathbf{q}, x}$, так как ее направление распространения совпадает с таковым для падающей волны.

Чтобы найти матрицы отражения и прохождения, запишем формально прохождение и отражение одной компоненты Фурье через структуру:

$$
\begin{gathered}
\mathbf{1}_{\mathbf{q}}^{(+)}+\tilde{\mathbf{R}}_{\mathbf{q}}^{(+)}=\hat{V} \tilde{\mathbf{T}}_{\mathbf{q}}^{(+)}, \\
\tilde{\mathbf{T}}_{\mathbf{q}}^{(-)}=\hat{V}\left(\mathbf{l}_{\mathbf{q}}^{(-)}+\tilde{\mathbf{R}}_{\mathbf{q}}^{(-)}\right) .
\end{gathered}
$$

Поскольку исходные векторы $\mathbf{R}_{\mathbf{q}}^{( \pm)}, \mathbf{T}_{\mathbf{q}}^{( \pm)}$из (3) имеют в два раза меньший размер, чем новые $\tilde{\mathbf{R}}_{\mathbf{q}}^{( \pm)}, \tilde{\mathbf{T}}_{\mathbf{q}}^{( \pm)}$, то системы (41), (42) достаточно для их однозначного определения. После нахождения векторов отражения и пропускания для всех поляризаций и фурье-компонент можно записать матрицу рассеяния и приступать непосредственно к процедуре $S$-квантования.

\section{4. Заключение}

Разработана процедура квантования электромагнитного поля в фотонных наноструктурах с трехмерной модуляцией диэлектрической проницаемости. Процедура квантования трехмерных структур осуществлена в ящике квантования с постановкой граничных условий $(S$-граничные условия), основанных на приравнивании амплитуд бегущих волн на противоположных сторонах ящика квантования, что соответствует приравниванию единице собственных векторов матрицы рассеяния ( $S$-матрицы). Получены выражения для полной $S$-матрицы в трехмерном случае, записанной через матрицы отражения и пропускания неоднородной структуры. Представлен метод, позволяющий нахождение матриц отражения и пропускания произвольных структур 
с трехмерной модуляцией диэлектрической проницаемости сведением к зависимости от двух декартовых координат, разделением структуры на параллельные слои и вычислением отдельных координатных и фурье-компонент. Предложенная процедура квантования электромагнитного поля в трехмерном случае позволяет реализовать прямой и самосогласованный метод вычисления спонтанной эмиссии излучателя, помещенного в трехмерные фотонные наноструктуры, избегая проблем со сходимостью при решении интегро-дифференциальных уравнений и значительно сокращая вычислительные ресурсы.

Авторы выражают благодарность Российскому научному фонду (грант № 16-12-10503) за финансовую поддержку.

\section{Список литературы}

[1] Р. Лоудон. Квантовая теория света (М., Мир, 1976) [Пер. с англ. R. Loudon. Quantum Theory of Light (Oxford University Press, 2000)].

[2] В.Л. Гинзбург. УФН, 140 (4), 687 (1983).

[3] E.M. Purcell. Phys. Rev., 69 (11), 681 (1946).

[4] В.П. Быков. ЖЭТФ, 35, 269 (1972).

[5] E. Yablonovitch. Phys. Rev. Lett., 58 (20), 2059 (1987).

[6] S. Brand, R.A. Abram, M.A. Kaliteevski. Phys. Rev. B, 75, 035102 (2007)

[7] F.J. García-de-Abajo. Rev. Mod. Phys., 79, 1267 (2007).

[8] C. Symonds, G. Lheureux , J.-P. Hugonin, J.-J. Greffet, J. Laverdant, G. Brucoli, A. Lemaitre, P. Senellart, J. Bellessa. Nano Lett., 13 (7), 3179 (2013).

[9] Л. Мандель, Э. Вольф. Оптическая когерентность и квантовая оптика (М., Физматлит, 2000.) [Пер. с англ. L. Mandel, E. Wolf. Optical coherence and quantum optics (Cambridge, University Press, 1995)].

[10] Л.Д. Ландау, Е.М. Лифшиц. Теоретическая физика. Т. 4. В.Б. Берестецкий, Е.М. Лифшиц, Л.П. Питаевский. Квантовая электродинамика. 4-е изд.(М., Физматлит, 2002).

[11] F. De Martini, M. Marrocco, P. Mataloni, L. Crescentini, R. Loudon. Phys. Rev. A, 43 (5), 2480 (1991).

[12] М.А. Калитеевский, В.А. Мазлин, К.А. Иванов, А.Р. Губайдуллин. Опт. и спектр., 119 (5), 810 (2015).

[13] M.S. Tomas, Z. Lenac. Phys. Rev. A, 56 (5), 4197 (1997).

[14] S.G. Tikhodeev, A.L. Yablonskii, E.A. Muljarov, N.A. Gippius, T. Ishihara. Phys. Rev. B, 66, 045102 (2002).

[15] М.А. Калитеевский, А.Р. Губайдуллин, К.А. Иванов, В.А. Мазлин. Опт. и спектр., 121 (3), 446 (2016).

[16] J.B. Pendry. J. Mod. Opt., 41, 209 (1994).

[17] М. Борн, Э. Вольф. Основы оптики (М., Наука, 1973).

[18] К.А. Иванов, В. В. Николаев, А.Р. Губайдуллин, М.А. Калитеевский. Опт. и спектр., 123 (4), 616 (2017).

\section{Quantization of an electromagnetic field in three-dimensional photonic structures based on the scattering matrix formalism ( $S$-quantization)}

\author{
K.A. Ivanov ${ }^{1}$, A.R. Gubaydullin ${ }^{1,3}$, M.A. Kaliteevski $i^{1,2,3}$ \\ 1 ITMO University, \\ 197101 St. Petersburg, Russia \\ 2 loffe Institute, \\ 194021 St. Petersburg, Russia \\ ${ }^{3}$ St. Petersburg Academic University, \\ 194021 St. Petersburg, Russia
}

\begin{abstract}
Based on the scattering matrix formalism, the method of quantization of the electromagnetic field in photonic nanostructures with three-dimensional modulation of the dielectric constant ( $S$-quantization in the three-dimensional case) is developed. Quantization is based on equating the eigenvalues of the scattering matrix to unity, which is equivalent to equating each other the set of fields, which are expanded into Fourier series, of the waves incidenting on the structure to the set of waves which travel away from the structure (outgoing waves). In the photon nanostructure, the spatial variation of the electromagnetic field of the modes is calculated on the basis of the $\tilde{R}$ and $\hat{T}$ matrices describing the reflection and transmission of the Fourier components through the structure. To calculate the reflection and the transmission coefficients of individual coordinate- and Fourier-components, the structure is divided into parallel layers, in which the dielectric constant varies in a two-dimensional coordinates. Using the Fourier transform, Maxwell's equations are written in the form of a matrix connecting the Fourier components of the electric field at the boundaries of neighboring layers. Based on the calculated vectors of reflection and transmission for all polarizations and Fourier components, a scattering matrix is formed for the entire structure and quantization is performed by equating the eigenvalues of the scattering matrix to unity. The developed method makes it possible to obtain spatial profiles of eigenmodes without solving a system of nonlinear integro-differential equations, and significantly reduces the computational resources necessary for calculating the probability of spontaneous emission in three-dimensional systems.
\end{abstract}

Редактор Л.В. Шаронова 